\title{
A Queueing Model for a Two-stage Stochastic Manufacturing System with Overlapping Operations
}

\author{
I. Van Nieuwenhuyse \\ Centre for Modelling and Simulation, European University College Brussels, Stormstraat 2, \\ 1000 Brussels, Belgium \\ inneke.vannieuwenhuyse@ehsal.be \\ N. Vandaele \\ Department of Applied Economics, University of Antwerp, Prinsstraat 13, 2000 Antwerp, \\ Belgium \\ nico.vandaele@ua.ac.be
}

\begin{abstract}
This is a postprint version of the article published in International Journal of Flexible Manufacturing Systems: Van Nieuwenhuyse, I., Vandaele, N. (2005). A queueing model for a two-stage stochastic manufacturing system with overlapping operations. International Journal of Flexible Manufacturing Systems, 17(3), 175-199. DOI 10.1007/s10696-006-8818-2. Available online at http://link.springer.com/.
\end{abstract}




\begin{abstract}
This paper presents analytical expressions for estimating average process batch flow times through a stochastic manufacturing system with overlapping operations. It is shown that the traditional queueing methodology cannot be directly applied to this setting, as the use of the overlapping operations principle causes the arrival process of sublots at the second stage to be a non-renewal process. An embedded queueing model is then proposed, which provides a tool to estimate the flow time reductions caused by the use of overlapping operations. Moreover, we provide expressions to estimate the production disruptions occurring at the second stage. The results of our research confirm the general intuition that the overlapping operations principle leads to less congestion, and hence a smoother flow of work through the system. On the other hand however, lot splitting inevitably requires more material handling on the shop floor. The expressions provided in this paper allow the quantification of the trade-off between these two effects, e.g., by gauging them within the scope of a cost model.
\end{abstract}

Keywords: lot splitting, overlapping operations, queueing theory 


\section{Introduction: Process batching versus transfer batch- ing}

The past decades have witnessed a surge in research efforts, aimed at modeling the performance of stochastic production or service systems by means of queueing theory (e.g., Karmarkar et al., 1985; Karmarkar, 1987; Lambrecht et al., 1998). While exact models are only available for a limited number of settings (like $\mathrm{M} / \mathrm{M} / 1$ or $\mathrm{M} / \mathrm{G} / 1$, see, e.g., Kleinrock, 1975), approximate models have been developed to estimate performance measures (like average flow times and WIP) under more general conditions, such as GI/G/1 or GI/G/m (Kramer and Lagenbach-Belz, 1967; Whitt, 1983; Whitt, 1993). These models are highly valued for their speed and ease of use in providing estimates of the performance indicators of interest, as opposed to simulation models (see Suri et al., 1993; Suri, 1998).

It is well-known that the average flow time of parts through a system is impacted by a range of managerial decisions, such as, e.g., the product mix being produced in the shop, the layout of the shop, and the batching policies used on the shopfloor. The impact of batching policies on flow times is particularly interesting to analyze: by setting batch sizes in a deliberate way, managers can obtain flow time improvements without a radical intervention in the system, and without large financial investments. Hence, setting batch sizes in a production system is an important control (see, e.g., Hopp and Spearman, 2000; Lambrecht et al., 1998; Benjaafar, 1996).

When studying the impact of batching policies, a distinction should be made between two types of batches: i.e., process batches and transfer batches. A process batch (also referred to as a production batch or production lot) is defined as the quantity of a product processed on a machine without interruption by other items (Kropp and Smunt, 1990). In multiple-product environments, the use of process batches is often unavoidable because of capacity considerations: to switch from one product type to the next, a setup or changeover time is necessary (e.g., to change fixtures or dies), which consumes part of the capacity of the machine. After a setup has been performed, a certain quantity of the product (the process batch size) can be produced. Hence, a process batch can also be defined as the quantity of a product produced between two consecutive setups.

The relationship between the process batch size and the average flow time has been thoroughly studied, and is convex (Karmarkar, 1987; Suri, 1998). Current queueing models are able to take into account the impact of the process batching policy (e.g., Karmarkar, 1985a 
and 1985b), and insight into the convex relationship has stirred the development of optimization procedures, aimed at determining the optimal process batch size for a given objective function (e.g., the weighted average flow time. See Lambrecht et al., 1996; Vandaele et al., 2003).

The term transfer batch on the other hand refers to the size of a subbatch of the process batch, moved after production on one machine to another operation or machine (Kropp and Smunt, 1990). The use of transfer batching (or lot splitting) is not caused by capacity considerations, but rather by flow considerations. Indeed, the use of transfer batch sizes smaller than the process batch size can reduce product flow times by smoothing workflow and minimizing congestion levels (e.g., Santos and Magazine, 1985; Benjaafar, 1996; Goldratt and Cox, 1984; Hopp et al., 1990; Umble and Srikanth, 1995). This is because of the mechanism of overlapping operations: by allowing transportation of partial batches to a downstream station, this station can already start processing these partial batches while work proceeds at the upstream station, thereby accelerating the progress of work through the production facility (see Graves and Kostreva, 1986; Jacobs and Bragg, 1988; Litchfield and Narasimhan, 2000).

The mechanism of overlapping operations provides most opportunities in systems with few (or no) delay servers ${ }^{1}$. Obviously, the excessive use of lot splitting may lead to capacity problems on this type of servers, as their utilization increases with the number of sublots used. This may cause congestion in front of the delay servers, which counteracts the benefits of the overlapping operations effect.

Given its positive impact on congestion levels, the use of lot splitting is quite common in practice, particularly in production settings where machines are located close together (such as in flowshop environments or manufacturing cells). Nevertheless, the impact of lot splitting in a stochastic setting has received little attention in the research literature. In current queueing models, the distinction between process batching and transfer batching is overlooked, as these models assume that products move between the machines in process batch sizes. This is also referred to as a lot-for-lot policy (see e.g., Van Nieuwenhuyse and Vandaele, 2004). A number of papers have studied the issue of lot splitting in stochastic settings by means of discrete-event simulation (see Wagner and Ragatz, 1994; Smunt et al., 1996; Jacobs and Bragg, 1988; Ruben and Mahmoodi, 1998). However, to the best of our

\footnotetext{
${ }^{1}$ Delay servers are resources for which the processing time of a sublot is independent of the sublot size (e.g., transportation resources are typically delay servers).
} 
knowledge, only a few studies (Bozer and Kim, 1996; Benjaafar, 1996) have analyzed the impact of transfer batching on flow times analytically. Moreover, the assumptions made in these papers are rather restrictive (e.g., Poisson arrival processes and/or Poisson service processes at the different stages of the system, regardless of the batching decisions).

In response to this hiatus, this paper presents an analytical model which enables one to capture the impact of lot splitting on flow times in a general two-stage stochastic setting; i.e., a manufacturing system that is characterized by a general arrival and service process and produces a single product family. As we show in sections 3.2 and 3.3, the analysis provides insights into the effect of lot splitting on the behavior of the second stage (e.g., the effect on production run start times and process batch makespan). As a starting point, section 2 briefly describes the structure and assumptions of the manufacturing system under study. It also outlines the difficulties arising in modeling the impact of overlapping operations by means of the queueing methodology. These hurdles provide the argument for the development of the embedded queueing model, the framework of which is described in detail in section 3. Section 4 presents the performance results of this embedded queueing model versus simulation results, and section 5 presents a cost model to analyze the trade-off between the flow time improvement and the increased material handling incurred on the shop floor. Finally, section 6 summarizes the conclusions.

\section{The two-stage manufacturing system with overlap- ping operations}

In this section, we first give an overview of the structure and assumptions of the system under study. Next, we discuss the inherent obstacles to the application of the queueing methodology.

\subsection{Structure and assumptions}

The setting we consider is a two-stage, stochastic manufacturing system which produces a single product family. It is an open system: production orders are launched into the system, undergo operations subsequently on the first and second stage, and leave the system after being processed. For simplicity, we assume that the size of a production order equals a process batch size. The interarrival times of process batches in the system, as well as the setup and processing times on the two servers, are generally distributed. Both stages consist 
of capacity servers (implying that the processing time of a sublot is dependent upon the size of the sublot), and are preceded by a buffer with FIFO queueing discipline.

Figure 1 illustrates the progress of two consecutive process batches, consisting of 4 sublots each, through the system. Upon arrival, a process batch may have to wait in front of the first stage before being processed (i.e., when this stage is still busy processing a previous process batch). When the server becomes idle, the setup is performed and the product units in the process batch are processed one by one. Products move between the two stages in transfer batches: it is assumed that the transfer batch size is a divisor of the process batch size, such that a process batch is split into an integer number of sublots. As soon as the transfer batch size is reached, the sublot is moved to the second stage (this is referred to in the literature as batch availability, e.g., Bukchin et al., 2002 and Santos and Magazine, 1985). It is important to note that, in our setting, the setup time on stage two is an attached setup (Chen and Steiner, 1998; Potts and Kovalyov, 2000), meaning that it can only start when the first transfer batch of the involved process batch has arrived in its input buffer. Hence, the first transfer batch of a process batch acts as a flag (Smunt et al., 1996): its arrival in front of the second stage authorizes the start of the setup, thereby causing the operations on stage two to partly overlap with the operations on stage one.

Figure 1. Flowchart of 2 process batches, consisting of 4 sublots, going through a 2-stage stochastic manufacturing system.

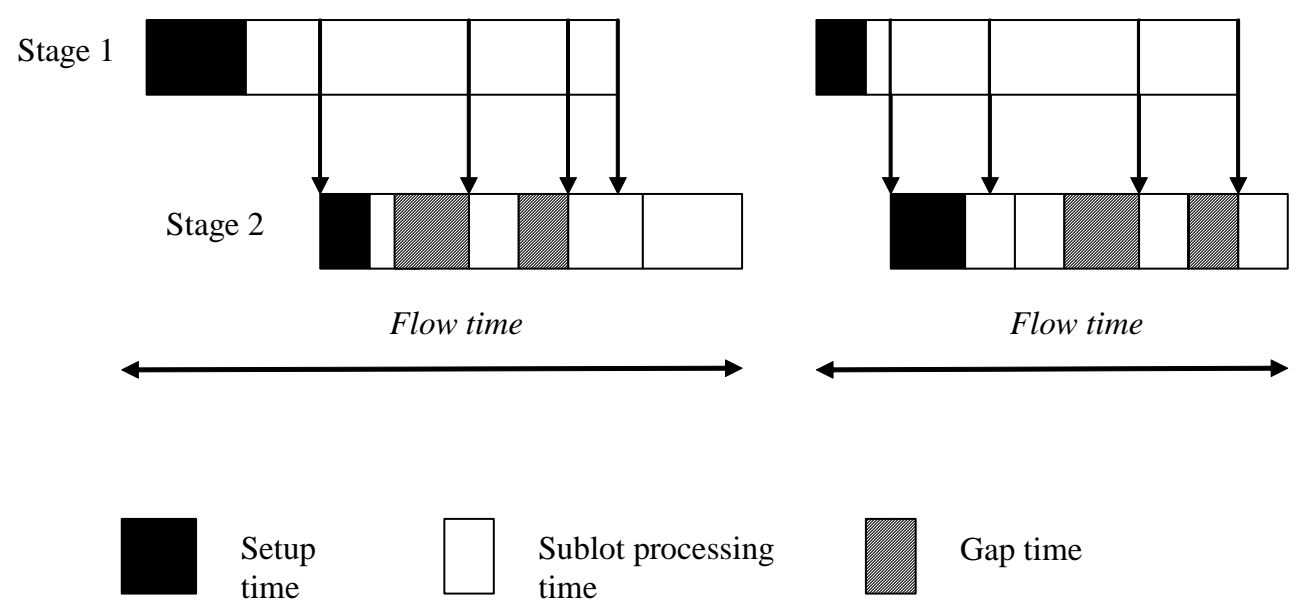

Upon arrival at the second stage, the flag may have to wait until the server becomes idle. As soon as it does, the setup for the process batch is performed and the flag is processed. The remaining sublots are processed as soon as they are finished on stage one, provided that 
stage two is available at that moment; otherwise, they will wait in the input buffer of the second stage.

The structure of the system described above implies that the second server may remain idle between the processing of two consecutive sublots, belonging to the same process batch. These idle times are referred to as gaps (Van Nieuwenhuyse, 2004; Van Nieuwenhuyse and Vandaele, 2004b). As illustrated in Figure 1, they occur whenever the second stage finishes processing a sublot before the next sublot is available from the first stage. In deterministic settings, gaps can be avoided by synchronizing the processing rates on the different machines in the shop (this is referred to as the no-idling assumption, e.g., Baker and Jia, 1993; Ramasesh et al., 2000); in stochastic settings however, gaps may occur even when the system is perfectly synchronized, due to the inherent variability in the setup and processing times at the different stages. The occurrence of gaps obviously leads to an increase in the average makespan of a process batch on stage two, without adding value to the product; hence, it represents a negative but unavoidable consequence of the use of lot splitting.

\subsection{Obstacles to the application of the queueing methodology}

Current open queueing models analyze the performance of a production network by means of the decomposition approach. In this approach, the network is decomposed into the different building blocks (the servers). Each server has its own specific arrival and processing characteristics. In a first step, the queue in front of each server is analyzed separately based on three different input parameters: i.e., parameters for the arrival process, the service process and the utilization rate of the server (see e.g., Whitt 1983; Whitt, 1994; Buzacott and Shantikumar, 1985; Suri et al., 1993 for more details). The different servers in the network are linked together by means of so-called linking equations, which ensure that the output stream of a particular server represents the input stream for the next server in the product's routing. Finally, the results are recomposed in order to determine the performance of the entire production system.

The decomposition approach is however based upon the critical assumption that the interarrival times and service times at each service center are both independent and identically distributed (IID), and that there is no correlation between interarrival and service times. Unfortunately, this assumption does not hold in a system with lot splitting. To substantiate this point, Figure 2 shows the arrival pattern for sublots at the second stage, for the example previously shown in Figure 1. Typically, the arrival process will exhibit 
a bursty pattern: as soon as the flag has arrived, the remaining sublots of the same production batch will arrive within rather short time intervals. Next, there will be a longer time interval before the next flag arrives. Consequently, the interarrival times of sublots are correlated, and no longer stem from the same probability distribution. As mentioned above, this violates one of the basic assumptions underlying the decomposition approach.

Figure 2. Flowchart with arrival pattern of sublots and flags.
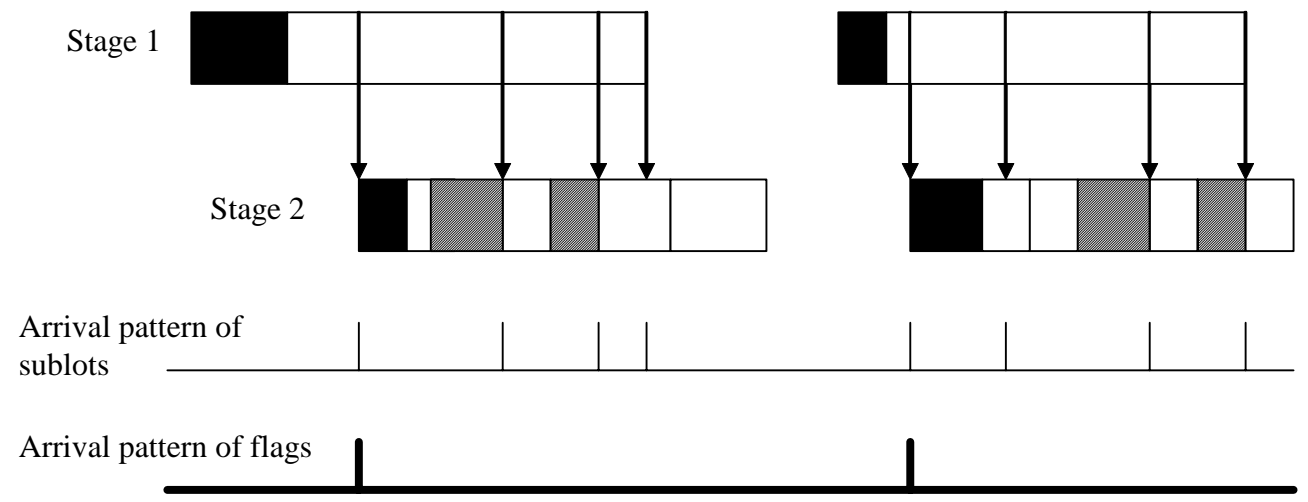

This issue may be circumvented by only considering the flags as entities moving through the network, instead of looking at individual transfer batches. Figure 2 illustrates the argument: indeed, the bursty pattern is caused by the fact that the interarrival times of the remaining sublots do not follow the same probability distribution as the interarrival times of the flags. When we only consider the flags as entities moving through the network, and ignore the remaining transfer batches, the bursty pattern disappears. The arrival process of flags in front of the second stage can be approximated by a renewal process, allowing us to estimate the average flow time of the flags by means of traditional queueing expressions. From the average flow time of the flags, we can then derive the average flow time of a process batch.

The resulting model will be referred to as the embedded queueing model. As explained in the next section, the proper application of this model calls for the development of appropriate approximations for the input parameters of the second stage. 


\section{3. $\quad$ Embedded queueing model}

In this section, we describe the structure of the embedded queueing model. Table 1 gives an overview of the notation. For any random variable $Z, E(Z)$ refers to the mean of this variable, $\operatorname{Var}(Z)$ to its variance, $\sigma(Z)$ to its standard deviation and $c_{Z}^{2}$ to its squared coefficient of variation (SCV).

Table 1. Overview of notation.

\begin{tabular}{ll}
\hline Parameters & \\
\hline$m$ & stage number index $(m=1,2)$ \\
$N$ & process batch size \\
$L$ & transfer batch size \\
$T$ & number of sublots in a process batch $\left(T=\frac{N}{L}\right)$ \\
$\lambda_{f, m}$ & average arrival rate of flags at stage $m$ \\
$\lambda_{P B, m}$ & average arrival rate of process batches at stage $m$ \\
$\rho_{m}$ & utilization of stage $m$ \\
\hline Random variables & \\
\hline$Y_{f, m}$ & interarrival time of flags at stage $m$ \\
$Y_{P B, m}$ & interarrival time of process batches at stage $m$ \\
$S U_{m}$ & setup time on stage $m$ \\
$x_{m}$ & unit processing time on stage $m$ \\
$X_{m}$ & transfer batch processing time on stage $m$ \\
$X_{m, i}$ & processing time of the $i$ th transfer batch of a process batch on stage $m$ \\
$G_{2}$ & total gap time for a process batch on stage 2 \\
$W_{f, m}$ & waiting time in queue of flags at stage $m$ \\
$P_{m}$ & process batch makespan at stage $m$ \\
$\tau_{f, 1}$ & interdeparture time of flags at stage 1 \\
$F_{P B}$ & flow time of a process batch through the system \\
$F_{f}$ & flow time of a flag through the system \\
\hline
\end{tabular}

\subsection{Model structure and assumptions}

As illustrated in Figure 2, the average flow time of a process batch through the system can be approximated by estimating the average flow time of a flag, and adding the average extra time elapsing on the second stage. This average extra time consists of the average total gap time occurring on the second stage, and the average processing time of the remaining $T-1$ transfer batches. This yields

$$
E\left(F_{P B}\right)=E\left(F_{f}\right)+(T-1) L E\left(x_{2}\right)+E\left(G_{2}\right)
$$


The average flow time of a flag consists of three components: the waiting time spent in queue at both stages, the setup time experienced at both stages, and the processing time of the flag itself. Hence

$$
E\left(F_{f}\right)=\sum_{m=1}^{2}\left[E\left(W_{f, m}\right)+E\left(S U_{m}\right)+L E\left(x_{m}\right)\right]
$$

Regarding each stage $m$ as a $G I / G / 1$ server, we expect that the average waiting time of a flag in front of each stage can be approximated by standard $G I / G / 1$ queueing expressions such as the Kraemer-Lagenbach-Belz expression (see Kraemer and Lagenbach-Belz 1967), which is in general given by

$$
E\left(W_{f, m}\right)_{a p p}= \begin{cases}=\frac{\rho_{m}^{2}\left(c_{Y_{f, m}}^{2}+c_{P_{m}}^{2}\right)}{2 \lambda_{f, m}\left(1-\rho_{m}\right)} \exp \left\{\frac{-2\left(1-\rho_{m}\right)\left(1-c_{Y_{f, m}}^{2}\right)^{2}}{3 \rho_{m}\left(c_{Y_{f, m}}^{2}+c_{P_{m}}^{2}\right)}\right\} & c_{Y_{f, m}}^{2} \leq 1 ; \\ =\frac{\rho_{m}^{2}\left(c_{Y_{f, m}}^{2}+c_{P_{m}}^{2}\right)}{2 \lambda_{f, m}\left(1-\rho_{m}\right)} & c_{Y_{f, m}}^{2}>1 .\end{cases}
$$

For the first stage, this yields no particular problems. As products arrive in process batches in front of stage 1, the average waiting time of a flag will equal the average waiting time of a process batch. Since setup and processing times on a server are assumed to be independent, we may write

$$
c_{P_{1}}^{2}=\frac{\operatorname{Var}\left(S U_{1}\right)+N \operatorname{Var}\left(x_{1}\right)}{\left[E\left(S U_{1}\right)+N E\left(x_{1}\right)\right]^{2}} .
$$

Moreover, $c_{Y_{f, m}}^{2}$ will be equal to the SCV of the interarrival times of process batches at stage 1 , and hence is given: $c_{Y_{f, 1}}^{2}=c_{Y_{P B, 1}}^{2}$. The utilization rate of stage 1 is given by

$$
\rho_{1}=\frac{E\left(S U_{1}\right)+N E\left(x_{1}\right)}{E\left(Y_{P B, 1}\right)} .
$$

Hence, $E\left(W_{f, 1}\right)_{a p p}$ can be readily calculated.

For the second stage however, only the utilization rate can be calculated in a straightforward manner:

$$
\rho_{2}=\frac{E\left(S U_{2}\right)+N E\left(x_{2}\right)}{E\left(Y_{P B, 2}\right)} .
$$

The expressions for $c_{Y_{f, 2}}^{2}$ and $c_{P_{2}}^{2}$ are not readily available: the interarrival time of flags at the second stage $\left(Y_{f, 2}\right)$ is determined by the interdeparture time of flags at the first stage (which we will denote by $\tau_{f, 1}$ ), and the makespan of a process batch at the second stage $\left(P_{2}\right)$ needs to include the total gap time. Consequently, estimating $E\left(W_{f, 2}\right)_{a p p}$ first requires us 
to develop appropriate approximations for these input parameters.

Approximations for $c_{Y_{f, 2}}^{2}$ and $c_{P_{2}}^{2}$ have been studied in detail in earlier research (Van Nieuwenhuyse 2004, Van Nieuwenhuyse and Vandaele 2004b, Van Nieuwenhuyse and Vandaele 2005). For ease of reference, we briefly summarize the results in sections 3.2 and 3.3.

\subsection{Analysis for $c_{P_{2}}^{2}$}

\subsubsection{Exact expressions and insights for $c_{P_{2}}^{2}$}

By definition, we can write

$$
c_{P_{2}}^{2}=\frac{\operatorname{Var}\left(P_{2}\right)}{E\left(P_{2}\right)^{2}}
$$

The random variable $P_{2}$ is given by

$$
P_{2}=S U_{2}+\sum_{i=1}^{T} X_{2, i}+G_{2}
$$

such that the exact expressions for $E\left(P_{2}\right)$ and $\operatorname{Var}\left(P_{2}\right)$ are given by

$$
E\left(P_{2}\right)=E\left(S U_{2}\right)+N * E\left(x_{2}\right)+E\left(G_{2}\right)
$$

and

$$
\begin{aligned}
\operatorname{Var}\left(P_{2}\right)= & \operatorname{Var}\left(S U_{2}\right)+N * \operatorname{Var}\left(x_{2}\right)+\operatorname{Var}\left(G_{2}\right) \\
& +2 \operatorname{Cov}\left(S U_{2}+\sum_{i=1}^{T} X_{2, i}, G_{2}\right) .
\end{aligned}
$$

Note that in this last expression, $\operatorname{Cov}\left(S U_{2}+\sum_{i=1}^{T} X_{2, i}, G_{2}\right) \leq 0$. Expressions (6) and (7) allow us to derive a number of intuitive insights about the impact of a lot splitting policy on the variability of $P_{2}$. If we let the subscript $L S$ refer to the use of a lot splitting policy, and $L F L$ to the use of a lot-for-lot policy, expressions (6) and (7) can be rewritten as:

$$
E\left(P_{2, L S}\right)=E\left(P_{2, L F L}\right)+E\left(G_{2}\right)
$$

and

$$
\operatorname{Var}\left(P_{2, L S}\right)=\operatorname{Var}\left(P_{2, L F L}\right)+\operatorname{Var}\left(G_{2}\right)+2 \operatorname{Cov}\left(S U_{2}+\sum_{i=1}^{T} X_{2, i}, G_{2}\right) .
$$

Hence, we have

$$
\begin{aligned}
& c_{P_{2, L F L}}^{2} \leq c_{P_{2, L S}}^{2} \\
\Leftrightarrow & \frac{\operatorname{Var}\left(P_{2, L F L}\right)}{\operatorname{Var}\left(P_{2, L F L}\right)+\operatorname{Var}\left(G_{2}\right)+2 \operatorname{Cov}\left(S U_{2}+\sum_{i=1}^{T} X_{2, i}, G_{2}\right)} \leq \frac{E\left(P_{2, L F L}\right)^{2}}{\left[E\left(P_{2, L F L}\right)+E\left(G_{2}\right)\right]^{2}} \leq 1 .
\end{aligned}
$$


The last inequality follows from the fact that $E\left(G_{2}\right)$ must be nonnegative. Condition (10) allows us to derive the following insights:

- In the presence of an outspoken upstream bottleneck, lot splitting will tend to increase the variability of process batch makespan at the second stage: A necessary condition for expression (10) to be valid, is that $\operatorname{Var}\left(G_{2}\right)+2 \operatorname{Cov}\left(S U_{2}+\right.$ $\left.\sum_{i=1}^{T} X_{2, i}, G_{2}\right) \geq 0$. This will very likely be the case in settings where the first stage is the bottleneck stage (i.e., where $E\left(x_{1}\right)>E\left(x_{2}\right)$ ): in that case, the behaviour of the gap time $G_{2}$ will be predominantly determined by the processing times on stage 1, implying that $\operatorname{Cov}\left(S U_{2}+\sum_{i=1}^{T} X_{2, i}, G_{2}\right)$ will be close to zero. Hence, the presence of an upstream bottleneck will tend to increase the variability of downstream process batch makespans when lot splitting is used.

- In the presence of an outspoken downstream bottleneck, the effect of lot splitting on the variability of process batch makespan is dampened: In settings where stage 2 is the outspoken bottleneck (i.e., where $E\left(x_{1}\right)<E\left(x_{2}\right)$ ), $G_{2}$ will be almost constant and equal to zero, such that $c_{P_{2, L F L}}^{2} \approx c_{P_{2, L S}}^{2}$.

- In the presence of no outspoken upstream or downstream bottleneck (i.e. the two stages are balanced), the use of lot splitting will tend to decrease the variability of process batch makespan on stage 2: A sufficient condition for expression (10) to be violated, is that $\operatorname{Var}\left(G_{2}\right)+2 \operatorname{Cov}\left(S U_{2}+\sum_{i=1}^{T} X_{2, i}, G_{2}\right)<0$. This is likely to be the case when the two stages are closely balanced (i.e., $E\left(x_{1}\right) \approx E\left(x_{2}\right)$ ), as then the covariance term usually attains a significantly negative value which outweighs the positive impact of $\operatorname{Var}\left(G_{2}\right)$.

In a general setting, the terms $E\left(G_{2}\right), \operatorname{Var}\left(G_{2}\right)$ and $\operatorname{Cov}\left(S U_{2}+\sum_{i=1}^{T} X_{2, i}, G_{2}\right)$ in expressions (6) and (7) cannot be exactly determined. Indeed, it can be shown (see Figure 1 for an intuitive illustration; a formal proof can be found in Van Nieuwenhuyse 2004) that the exact expression for $G_{2}$ is given by

$$
G_{2}=\max [K(2), \ldots, K(T), 0]
$$

with $K(i)=\sum_{k=2}^{i} X_{1, k}-\left(W_{f, 2}+S U_{2}+\sum_{j=1}^{i-1} X_{2, j}\right)$ for $(i=2, \ldots, T)$. Consequently, the total gap time $G_{2}$ is a random variable consisting of the maximum of $T-1$ correlated random variables $K(t)$ (with $t=2, \ldots, T$ ) and a constant (zero). 
Interestingly, in the corresponding deterministic setting, $X_{1}, X_{2}$ and $S U_{2}$ are constants instead of random variables, and $W_{f, 2}$ will equal 0 (provided that the system is stable). Hence, in a system without variability, expression (11) reduces to:

$$
G_{2, \text { det }}=\max \left[(T-1)\left(X_{1}-X_{2}\right)-S U_{2}, 0\right]
$$

which confirms earlier results for the calculation of $G_{2}$ in a deterministic setting (see Van Nieuwenhuyse and Vandaele, 2004a for an extensive discussion).

In a general stochastic setting, the probability distribution of $G_{2}$ is analytically intractable. Hence, approximating $c_{P_{2}}^{2}$ requires approximations for $E\left(G_{2}\right), \operatorname{Var}\left(G_{2}\right)$ and $\operatorname{Cov}\left(S U_{2}+\sum_{i=1}^{T} X_{2, i}, G_{2}\right)$. These approximations are further discussed in the next subsection.

\subsubsection{Approximation for $c_{P_{2}}^{2}$}

Based upon results from earlier research (Van Nieuwenhuyse and Vandaele 2005, Van Nieuwenhuyse 2004), we propose to approximate the total gap time $G_{2}$ by

$$
G_{2, a p p}=\max [Z, 0]
$$

with $Z$ a normally distributed random variable given by

$$
Z=\sum_{k=2}^{T} X_{1, k}-\left(S U_{2}+\sum_{j=1}^{T-1} X_{2, j}\right)
$$

Simulation experiments for a wide variety of settings (see Van Nieuwenhuyse 2004 for an extensive discussion) have indeed shown that the probability distribution of $G_{2}$ can be approximated by a zero-inflated normal distribution. It is particularly useful to confront the proposed approximation with the theoretically exact expression for $G_{2}$ in expression (11). This reveals that our approximation makes two implicit assumptions. Firstly, the approximation ignores the negative relationship between the waiting time of the flag in front of stage $2\left(W_{f, 2}\right)$ and the gap time occurring on stage $2\left(G_{2}\right)$. Consequently, our approximation will tend to overestimate the average gap time, particularly in settings where the utilization of stage 2 is high. However, we may expect that the magnitude of the resulting relative error on $P_{2}$ will stay at a reasonable level: large values for $W_{f, 2}$ are bound to occur when the processing rate of stage 2 is relatively low, implying that in these settings gap times will constitute a very small (or even negligible) part of the process batch makespan. 
Moreover, expression (11) reveals that it suffices to have a positive value for any arbitrary $K(i)(i=2, \ldots, T)$ in order to have a positive gap time on stage 2 , while our approximation only takes into account the impact of $K(T)$. We may expect that this simplification will have little effect on the performance of our approximation, as long as $E\left(x_{1}\right)$ is significantly larger than $E\left(x_{2}\right)$ (i.e., when the system is unbalanced, with the bottleneck on the first machine). In other settings, this simplification might lead to more pronounced relative errors on $P_{2}$.

The proposed approximation is consistent with the exact results for the calculation of $G_{2}$ in a deterministic setting. Note that $E(Z)$ and $\operatorname{Var}(Z)$ are given by

$$
\begin{aligned}
& E(Z)=(T-1) *\left[E\left(X_{1}\right)-E\left(X_{2}\right)\right]-E\left(S U_{2}\right), \\
& \operatorname{Var}(Z)=(T-1) *\left[\operatorname{Var}\left(X_{1}\right)+\operatorname{Var}\left(X_{2}\right)\right]+\operatorname{Var}\left(S U_{2}\right) .
\end{aligned}
$$

In the deterministic setting, $X_{1}, X_{2}$ and $S U_{2}$ are constants such that $\operatorname{Var}\left(X_{1}\right)=\operatorname{Var}\left(X_{2}\right)=$ $\operatorname{Var}\left(S U_{2}\right)=0$. Hence, both expressions (14) and (13) reduce to a constant:

$$
\begin{gathered}
Z_{\text {det }}=(T-1)\left[\left(X_{1}\right)-\left(X_{2}\right)\right]-\left(S U_{2}\right), \\
G_{2, \text { det }}=\max \left[Z_{\text {det }}, 0\right] .
\end{gathered}
$$

which confirms expression (12). Note that $E(Z)$ (the average of the underlying normal distribution $Z$ ) in the approximation for the stochastic setting coincides with $Z_{\text {det }}$ for the corresponding deterministic case.

For any zero-inflated normal variable $V^{\prime}=\max [0, V]$ for which $E(V)$ and $\operatorname{Var}(V)$ are known, the first moment $E\left(V^{\prime}\right)$ and second moment $E\left(V^{\prime 2}\right)$ can be determined numerically (see Blumenfeld, 2001). For ease of reference, the expressions are outlined in Appendix 1. Hence, using the zero-infated normal approximation proposed in expressions (13) and (14), we can approximate $E\left(G_{2}\right)$ and $\operatorname{Var}\left(G_{2}\right)$ as follows:

$$
\begin{aligned}
E\left(G_{2, \text { app }}\right)= & \frac{E(Z)}{2}\left(1+\operatorname{Er} f\left[\frac{E(Z)}{\sqrt{2 \operatorname{Var}(Z)}}\right]\right)+\operatorname{Exp}\left\{\frac{-E(Z)^{2}}{2 \operatorname{Var}(Z)}\right\} \sqrt{\frac{\operatorname{Var}(Z)}{2 \pi}}, \\
\operatorname{Var}\left(G_{2, \text { app }}\right)= & \operatorname{Exp}\left\{\frac{-E(Z)^{2}}{2 \operatorname{Var}(Z)}\right\} E(Z) \sqrt{\frac{\operatorname{Var}(Z)}{2 \pi}}+\frac{E(Z)^{2}+\operatorname{Var}(Z)}{2}\left(1+\operatorname{Erf}\left[\frac{E(Z)}{\sqrt{2 \operatorname{Var}(Z)}}\right]\right) \\
& -\left(E\left(G_{2}\right)_{\text {app }}\right)^{2} .
\end{aligned}
$$

Let's now turn to $\operatorname{Cov}\left(S U_{2}+\sum_{i=1}^{T} X_{2, i}, G_{2}\right)$. It is clear that $X_{2, T}$ is independent of $G_{2}$, 
such that we can write

$$
\operatorname{Cov}\left(S U_{2}+\sum_{i=1}^{T} X_{2, i}, G_{2}\right)=\operatorname{Cov}\left(S U_{2}+\sum_{i=1}^{T-1} X_{2, i}, G_{2}\right)
$$

We propose the following approximation:

$$
\begin{aligned}
& \operatorname{Cov}\left(S U_{2}+\sum_{i=1}^{T} X_{2, i}, G_{2}\right) \\
= & \operatorname{Cov}\left(S U_{2}+\sum_{i=1}^{T-1} X_{2, i}, G_{2, a p p}\right) \\
\approx & \operatorname{Cov}\left(U, G_{2, a p p}\right) \\
\approx & \operatorname{Cov}(U, \max [Z, 0])
\end{aligned}
$$

assuming a normal distribution for $U=S U_{2}+\sum_{i=1}^{T-1} X_{2, i}$, and the zero-inflated normal distribution for $G_{2, a p p}=\max [Z, 0]$ where $Z$ is given by expression (14).

For any two correlated normal random variables $U$ and $Z, \operatorname{Cov}(U, \max [Z, 0])$ can be numerically determined when the average and variance of both $U$ and $Z$ are known, and the correlation coefficient $\rho_{U, Z}$ is known. The general approach is outlined in Appendix 2. In our case, $E(Z)$ and $\operatorname{Var}(Z)$ are given in expression (15) above, and $E(U)$ and $\operatorname{Var}(U)$ are determined by

$$
\begin{gathered}
E(U)=E\left(S U_{2}\right)+(T-1) E\left(X_{2}\right), \\
\operatorname{Var}(U)=\operatorname{Var}\left(S U_{2}\right)+(T-1) \operatorname{Var}\left(X_{2}\right) .
\end{gathered}
$$

The correlation coefficient $\rho_{U, Z}$ equals

$$
\rho_{U, Z}=\frac{-\operatorname{Var}\left(S U_{2}\right)-(T-1) \operatorname{Var}\left(X_{2}\right)}{\sigma_{U} \sigma_{Z}} .
$$

Hence, using the approach in Appendix 2, $\operatorname{Cov}(U, \max [Z, 0])$ can be obtained.

Using expressions (16) and (17) in expressions (6) and (7) yields the following approximations for $E\left(P_{2, a p p}\right)$ and $\operatorname{Var}\left(P_{2, a p p}\right)$ :

$$
\begin{gathered}
E\left(P_{2, a p p}\right)=E\left(S U_{2}\right)+N E\left(x_{2}\right)+E\left(G_{2, a p p}\right), \\
\operatorname{Var}\left(P_{2, a p p}\right)=\operatorname{Var}\left(S U_{2}\right)+N \operatorname{Var}\left(x_{2}\right)+\operatorname{Var}\left(G_{2, a p p}\right)+2 \operatorname{Cov}\left(U, G_{2, a p p}\right) .
\end{gathered}
$$

Finally, we obtain the following approximation for $c_{P_{2}}^{2}$ :

$$
c_{P_{2, a p p}}^{2}=\frac{\operatorname{Var}\left(P_{2, a p p}\right)}{\left[E\left(P_{2, a p p}\right)\right]^{2}} .
$$




\subsection{Analysis for $c_{Y_{f, 2}}^{2}$}

\subsubsection{Exact expressions and insights for $c_{Y_{f, 2}}^{2}$}

From the structure of the system, it is clear that $c_{Y_{f, 2}}^{2}$ equals $c_{\tau_{f, 1}}^{2}$ :

$$
c_{Y_{f, 2}}^{2}=c_{\tau_{f, 1}}^{2}=\frac{\operatorname{Var}\left(\tau_{f, 1}\right)}{E\left(\tau_{f, 1}\right)^{2}} .
$$

In a stable system, the average interdeparture time of flags $E\left(\tau_{f, 1}\right)$ will equal the average interarrival time of flags $E\left(Y_{f, 1}\right)$, and hence equals the average interarrival time of process batches in front of stage 1 :

$$
E\left(\tau_{f, 1}\right)=E\left(Y_{f, 1}\right)=E\left(Y_{P B, 1}\right) .
$$

The expression for $\operatorname{Var}\left(\tau_{f, 1}\right)$ is less straightforward. To illustrate the analysis, Figure 3 shows a flowchart of two process batches (process batch $n$ and process batch $(n+1)$, each consisting of 3 sublots, going through the first stage of the system. The vertical arrows at the top indicate the arrival times of the flags in front of the stage; the vertical arrows at the bottom denote the departure times of the consecutive flags leaving the stage.

Figure 3. Flowchart of two arbitrary process batches $n$ and $(n+1)$ going through stage 1
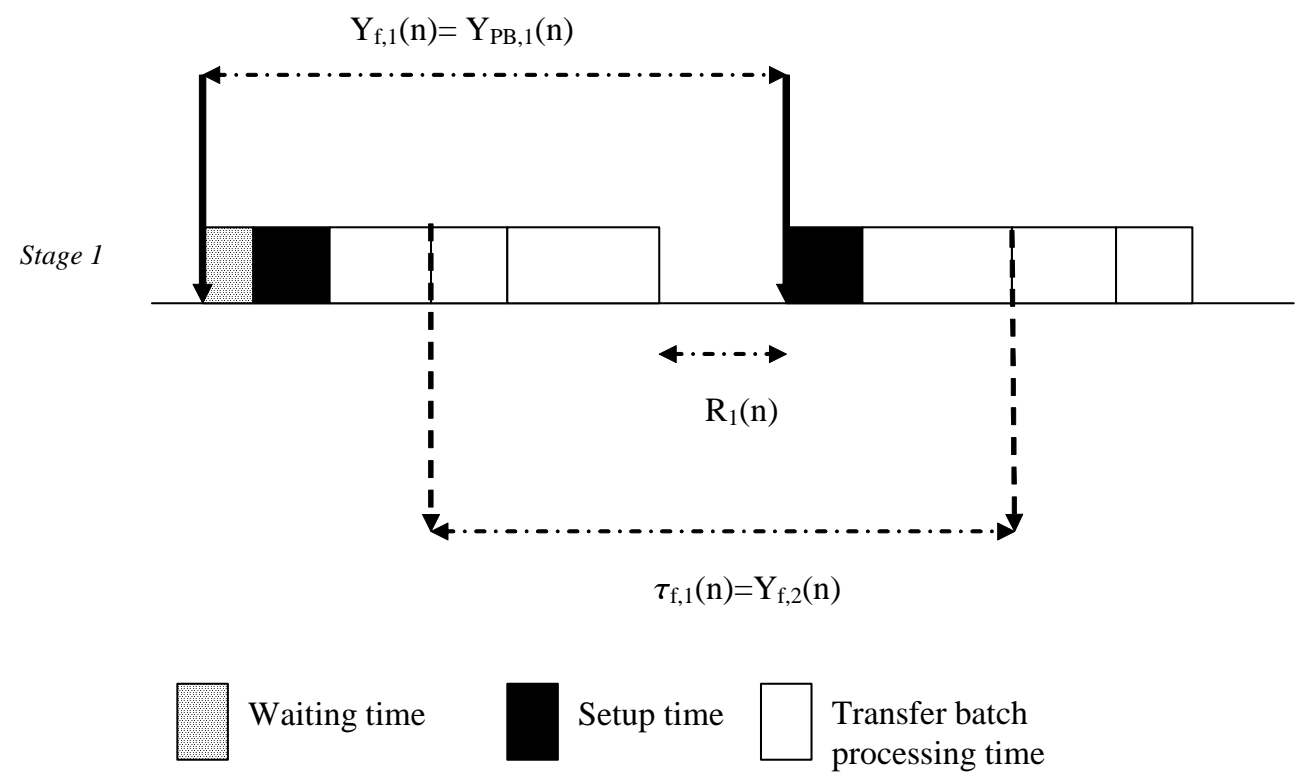

From Figure 3, we can derive the following relationship for the interdeparture time between flag $n$ and flag $(n+1)$ at stage 1 :

$$
\tau_{f, 1}(n)=\sum_{i=2}^{T} X_{1, i}(n)+R_{1}(n)+S U_{1}(n+1)+X_{1,1}(n+1) .
$$


In this expression, $R_{1}(n)$ refers to the idle time that may occur between the processing of consecutive process batches at stage 1:

$$
R_{1}(n)=\max \left[0, Y_{P B, 1}(n)-W_{f, 1}(n)-S U_{1}(n)-\sum_{i=1}^{T} X_{1, i}(n)\right] .
$$

As the transfer batch processing times are IID distributed and independent of the setup times, it follows from expression (20) that:

$$
\operatorname{Var}\left(\tau_{f, 1}(n)\right)=\operatorname{Var}\left(S U_{1}\right)+T \operatorname{Var}\left(X_{1}\right)+\operatorname{Var}\left(R_{1}(n)\right)+2 \operatorname{Cov}\left[\sum_{i=2}^{T} X_{1, i}(n), R_{1}(n)\right]
$$

This expression reveals an interesting relationship between the departure process of flags with a lot splitting policy, and the departure process of process batches with a lot-for-lot policy. Note that, with a lot-for-lot policy, the variance of the interdeparture time between the $n$th and the $(n+1)$ th process batch at stage $1\left(\operatorname{Var}\left[\tau_{P B, 1}(n)\right]\right)$ is given by:

$$
\operatorname{Var}\left(\tau_{P B, 1}(n)\right)=\operatorname{Var}\left(S U_{1}\right)+T \operatorname{Var}\left(X_{1}\right)+\operatorname{Var}\left(R_{1}(n)\right)
$$

such that expression (22) reduces to:

$$
\operatorname{Var}\left(\tau_{f, 1}(n)\right)=\operatorname{Var}\left(\tau_{P B, 1}(n)\right)+2 \operatorname{Cov}\left[\sum_{i=2}^{T} X_{1, i}(n), R_{1}(n)\right]
$$

or, in general:

$$
\operatorname{Var}\left(\tau_{f, 1}\right)=\operatorname{Var}\left(\tau_{P B, 1}\right)+2 \operatorname{Cov}\left[\sum_{i=2}^{T} X_{1, i}, R_{1}\right] .
$$

This allows to derive the following insights:

- Lot splitting improves the reliability of production run start times at the second stage of the system: As $\operatorname{Cov}\left[\sum_{i=2}^{T} X_{1, i}, R_{1}\right] \leq 0$ (see van Nieuwenhuyse 2004, Van Nieuwenhuyse en Vandaele 2004b for a formal proof), it evidently follows that $c_{\tau_{f, 1}}^{2} \leq c_{\tau_{P B, 1}}^{2}$, i.e. that the variability of the interdeparture times of flags under a lot splitting policy is smaller than or equal to the variability of the interdeparture times of process batches under a lot-for-lot policy. This observation provides additional rationale for the use of the lot splitting policy in manufacturing systems: indeed, it implies that the use of lot splitting improves the reliability of production run start times at the second stage. 
- The size of the improvement depends on the utilization of the first stage: Indeed, a high utilization at the first stage may undermine the positive effects of lot splitting. When the first stage is higly utilized (e.g. when it is the bottleneck operation), $R_{1} \rightarrow 0$ and $\operatorname{Cov}\left[\sum_{i=2}^{T} X_{1, i}, R_{1}\right] \rightarrow 0$, such that $c_{\tau_{f, 1}}^{2} \rightarrow c_{\tau_{P B, 1}}^{2}$. In this case, the reliability of production run start times is the same for the lot splitting policy and the lot-for-lot policy.

The exact expression for $\operatorname{Var}\left(\tau_{P B, 1}\right)$ has been derived by Marshall (1968):

$$
\begin{aligned}
\operatorname{Var}\left(\tau_{P B, 1}\right)= & 2\left(\operatorname{Var}\left[S U_{1}\right)+T \operatorname{Var}\left(X_{1}\right]\right)+\operatorname{Var}\left(Y_{P B, 1}\right) \\
& -2 E\left(W_{P B, 1}\right)\left(E\left(Y_{P B, 1}\right)-E\left(S U_{1}\right)-T E\left(X_{1}\right)\right)
\end{aligned}
$$

Unfortunately, in a general setting, both $\operatorname{Cov}\left[\sum_{i=2}^{T} X_{1, i}, R_{1}\right]$ in expression $(23)$ and $E\left(W_{P B, 1}\right)$ in expression (24) are analytically intractable. Moreover, $\operatorname{Cov}\left[\sum_{i=2}^{T} X_{1, i}, R_{1}\right]$ does not equal 0 : recall from expression $(21)$ that $R_{1}=\max \left[0, Y_{1}-W_{f, 1}-S U_{1}-\sum_{i=1}^{T} X_{1, i}\right]$, such that $R_{1}$ and $\sum_{i=2}^{T} X_{1, i}$ are clearly not independent. Hence, approximating $\operatorname{Var}\left(\tau_{f, 1}\right)$ requires us to approximate $\operatorname{Var}\left(\tau_{P B, 1}\right)$ and $\operatorname{Cov}\left[\sum_{i=2}^{T} X_{1, i}, R_{1}\right]$.

\subsubsection{Approximation for $c_{Y_{f, 2}}^{2}$}

From expression (23), it is clear that an approximation for $\operatorname{Var}\left(\tau_{f, 1}\right)$ can be obtained by approximating its two main components: $\operatorname{Var}\left(\tau_{P B, 1}\right)$ and $\operatorname{Cov}\left[\sum_{i=2}^{T} X_{1, i}, R_{1}\right]$. As $E\left(W_{P B, 1}\right)=$ $E\left(W_{f, 1}\right)$, an approximation for $\operatorname{Var}\left(\tau_{P B, 1}\right)$ is directly obtained using expression $(24)$ :

$$
\begin{aligned}
\operatorname{Var}\left(\tau_{P B, 1}\right)_{a p p}= & 2\left(\operatorname{Var}\left[S U_{1}\right)+T \operatorname{Var}\left(X_{1}\right]\right)+\operatorname{Var}\left(Y_{P B, 1}\right) \\
& -2 E\left(W_{P B, 1}\right)_{a p p}\left(E\left(Y_{P B, 1}\right)-E\left(S U_{1}\right)-T E\left(X_{1}\right)\right) .
\end{aligned}
$$

and calculating $E\left(W_{P B, 1}\right)_{a p p}=E\left(W_{f, 1}\right)_{a p p}$ by the formula of Kramer-Lagenbach Belz in expression (3).

Hence, we can restrict ourselves to developing an approximation for $\operatorname{Cov}\left[\sum_{i=2}^{T} X_{1, i}, R_{1}\right]$. Previous research (see Van Nieuwenhuyse and Vandaele 2004b, Van Nieuwenhuyse and Vandaele 2006 for details) has shown that this covariance may be adequately approximated by the following:

$$
\operatorname{Cov}\left[\sum_{i=2}^{T} X_{1, i}, R_{1}\right]_{a p p}=\left(1-\rho_{1}^{2}\right) \operatorname{Cov}\left[V, R_{1, a p p}\right]
$$

with

$$
V=\sum_{i=2}^{T} X_{1, i}
$$




$$
\begin{gathered}
R_{1, a p p}=\max [0, M] \\
M=Y_{P B, 1}-S U_{1}-\sum_{i=1}^{T} X_{1, i}
\end{gathered}
$$

and assuming normal probability distributions for both $V$ and $M$. Note that $R_{1, \text { app }}$ differs from the theoretically exact expression for $R_{1}$ (see expression $(21)$ ), as the impact of $W_{f, 1}$ is ignored. From expression (21), it is clear that as $W_{f, 1}$ increases, $R_{1}$ will approach zero and consequently $\operatorname{Cov}\left[\sum_{i=2}^{T} X_{1, i}, R_{1}\right]$ will drop to zero (see also the discussion in section 3.3.1 above). In expression (26), we capture this behavior by including the factor $\left(1-\rho_{1}^{2}\right)$ in the approximation: as $\rho_{1} \rightarrow 1, \operatorname{Cov}\left[\sum_{i=2}^{T} X_{1, i}, R_{1}\right]_{a p p}$ indeed approaches 0 , which is what we would expect.

Also note that the assumption of a normal distribution for $M$ implies a zero-inflated normal probability distribution for $R_{1, a p p}$. As the average and variance of both $V$ and $M$ are known:

$$
\begin{gathered}
E(V)=(T-1) E\left(X_{1}\right), \\
E(M)=E\left(Y_{f, 1}\right)-\left[E\left(S U_{1}\right)+T E\left(X_{1}\right)\right], \\
\operatorname{Var}(V)=(T-1) \operatorname{Var}\left(X_{1}\right), \\
\operatorname{Var}(M)=\operatorname{Var}\left(Y_{f, 1}\right)+\operatorname{Var}\left(S U_{1}\right)+T \operatorname{Var}\left(X_{1}\right),
\end{gathered}
$$

and the correlation between $\mathrm{V}$ and $\mathrm{M}$ is given by:

$$
\rho_{V, M}=\frac{-\operatorname{Var}(V)}{\sigma_{V} \sigma_{M}}
$$

we can calculate $\operatorname{Cov}\left[V, R_{1, a p p}\right]$ numerically by applying the approach discussed in Appendix 2. Combining expressions (18), (19), (23), (25) and (26), we get the following approximation for $c_{Y_{f, 2}}^{2}$ :

$$
c_{Y_{f, 2}}^{2}=c_{\tau_{f, 1}}^{2}=\frac{\operatorname{Var}\left(\tau_{P B, 1}\right)_{a p p}}{\left[E\left(\tau_{P B, 1}\right)\right]^{2}}+2\left(1-\rho_{1}^{2}\right) \frac{\operatorname{Cov}\left[V, R_{1, a p p}\right]}{E\left[Y_{P B, 1}\right]^{2}} .
$$

\section{Performance of the embedded queueing model}

In this section, we will test the performance of the embedded queueing model in estimating $E\left[F_{P B}\right]$ by means of discrete-event simulation results. To this end, we study two settings: in the first setting (Case 1), the first stage is the outspoken bottleneck, whereas in the second setting (Case 2), the two stages are perfectly balanced. Consequently, the gap times occurring in Case 2 are solely due to the variability present in the system. 


\subsection{Simulation experiment}

Table 2 gives an overview of the input parameters for the two cases. Both cases were evaluated at three different utilization rates for stage $1: \rho_{1}=30 \%, 60 \%$ and $90 \%$. The random numbers for interarrival times, setup and processing times in the simulation experiment were all drawn from gamma distributions ${ }^{2}$. The runlength of the simulation was equal to 100000 process batches, of which the first 20000 were considered to be part of the warm-up period.

Table 2. Input parameters for Case 1 and Case 2.

\begin{tabular}{l|ll} 
& Case 1 & Case 2 \\
\hline \hline$N$ & 30 & 30 \\
$T$ & $1,2,3,5,6,10,15,30$ & $1,2,3,5,6,10,15,30$ \\
$E\left[S U_{1}\right]$ & 2 & 2 \\
$E\left[S U_{2}\right]$ & 2 & 2 \\
$E\left[x_{1}\right]$ & 1 & 1 \\
$E\left[x_{2}\right]$ & 0.2 & 1 \\
$E\left[x_{1}\right]$ & $30 \%, 60 \%$ or $90 \%$ & $30 \%, 60 \%$ or $90 \%$ \\
$\rho_{1}$ & 0.75 & 0.75 \\
$c_{S U_{1}}^{2}$ & 0.75 & 0.75 \\
$c_{S U_{2}}^{2}$ & 0.5 & 0.5 \\
$c_{x_{1}}^{2}$ & 0.5 & 0.5 \\
$c_{x_{2}}^{2}$ & 0.75 & 0.75 \\
$c_{Y_{P B, 1}}^{2}$ &
\end{tabular}

\subsection{Results}

Table 3 compares the resulting estimates for $E\left(F_{P B}\right)$ for Case 1 and Case 2, showing the percentage deviation of the queueing model $\left(E\left(F_{P B}\right)_{Q}\right)$ with respect to the simulation results $\left(E\left(F_{P B}\right)_{S}\right)$. The table reveals that the embedded queueing model tends to overestimate the simulation results. This is due to the fact that we have ignored $W_{f, 2}$ in our expression for $G_{2, a p p}$, leading to an overestimation of the average gap time (as discussed in section 3.2.2). However, as expected, the resulting relative error on $E\left(F_{P B}\right)$ stays at a reasonable level, particularly in Case 1. Indeed, in this case, the first stage is the outspoken bottleneck, such that the second stage is lowly utilized (indeed, note that even when $\rho_{1}=90 \%, \rho_{2}$ only

\footnotetext{
${ }^{2}$ This distribution was chosen because of its positive skewness, which is a desirable characteristic in particular for the setup and processing time components. Other suitable candidates would have been a lognormal distribution, or a $\operatorname{beta}(\alpha 1, \alpha 2)$ distribution with $1<\alpha 1<\alpha 2$. For the interarrival times, other probability distributions could have been considered.
} 
amounts to $22.5 \%$ ) and the impact of $W_{f, 2}$ on $G_{2}$ is negligible. In Case 2, the relative errors on $E\left(F_{P B}\right)$ are more pronounced: as the two stages are perfectly balanced, $\rho_{1}=\rho_{2}$ for all settings such that $W_{f, 2}$ now has a more significant impact on $G_{2}$. The relative error on $E\left(F_{P B}\right)$ in Case 2 is most pronounced at moderate utilization levels (e.g., $\rho_{1}=\rho_{2}=60 \%$ ); at high utilization levels (e.g., $\left.\rho_{1}=\rho_{2}=90 \%\right), E\left(F_{P B}\right)$ is largely determined by the waiting times of the flags in front of both stages, so though we may significantly overestimate $E\left(G_{2}\right)$ in absolute terms, the relative error on $E\left(F_{P B}\right)$ will be small.

On the whole, we may conclude that the performance of the embedded queueing model

Table 3. Results for $E\left(F_{P B}\right)_{Q}$ and $E\left(F_{P B}\right)_{S}$, for Case 1 and Case 2.

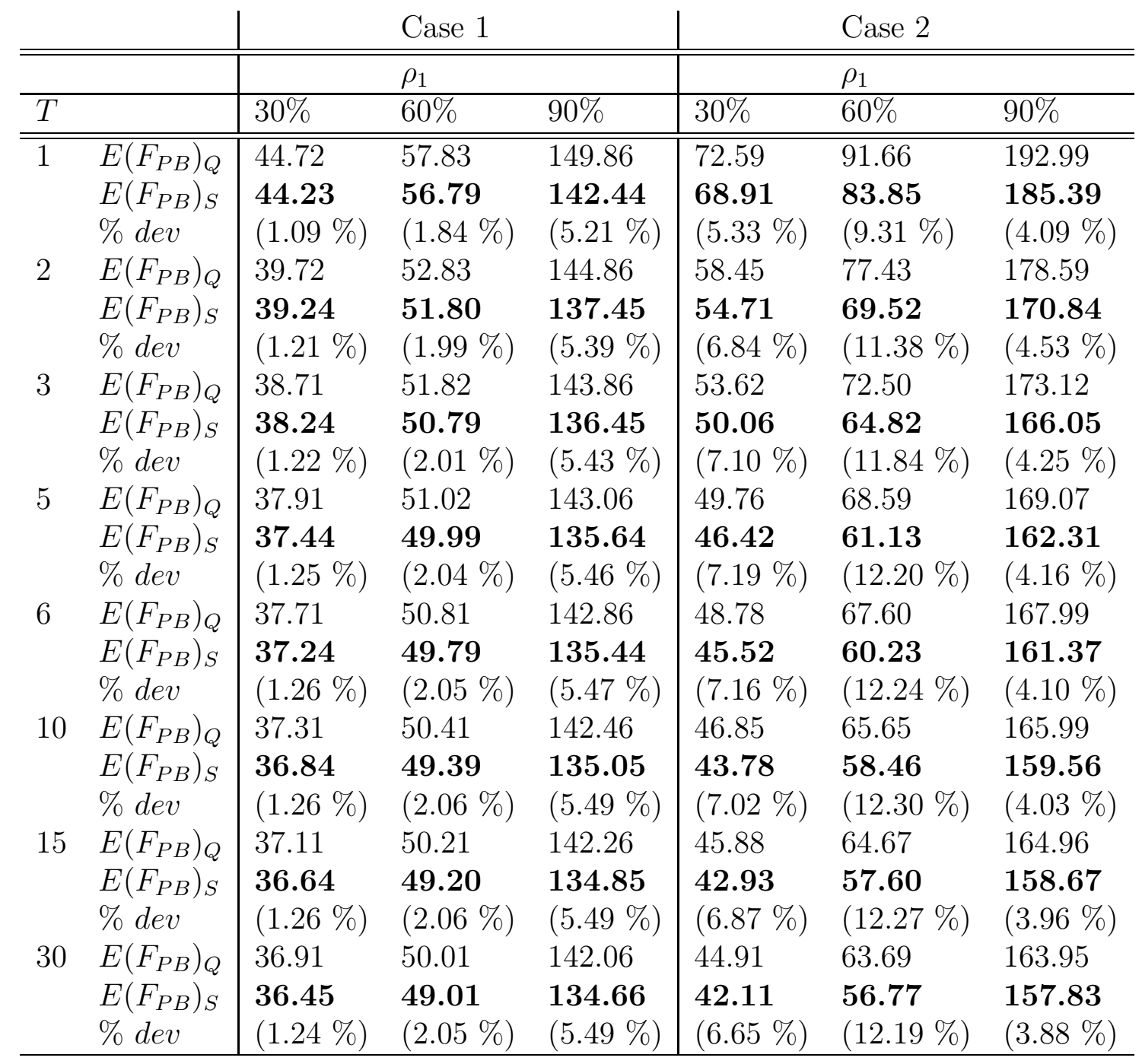

is satisfactory: the observed relative errors on $E\left(F_{P B}\right)$ are of the same order of magnitude for the lot-for-lot policy $(T=1)$ and the different lot splitting policies $(T>1)$, in all set- 
tings. Moreover, both $E\left(F_{P B}\right)_{Q}$ and $E\left(F_{P B}\right)_{S}$ are convex and continuously decreasing in the number of sublots, implying that the model adequately captures the impact of overlapping operations. The results confirm the general intuition that the use of lot splitting has a positive impact on $E\left(F_{P B}\right)$; however, it is clear that this impact is most pronounced at low values of $T$. As the number of sublots $T$ increases, the marginal improvement in $E\left(F_{P B}\right)$ becomes almost negligible.

\section{Economic evaluation: cost model}

In this section, we study the use of lot splitting in a two-stage stochastic system from a cost perspective, by focusing on two cost components: inventory holding costs and material handling $\operatorname{costs}^{3}$. Indeed, while lot splitting leads to lower average lead times (and, hence, lower average inventory in the system), it entails a larger number of material movements on the shop floor. In what follows, we study the trade-off between these two cost types in more detail, and illustrate it by means of an example.

\subsection{Model development and insights}

Focusing on inventory holding costs and material handling costs, the average total costs in a two-stage system can be written as:

$$
E\left[\text { Cost }_{\text {tot }}\right]=E\left[\text { Cost }_{\text {inv }}\right]+E\left[\text { Cost }_{\text {trans }}\right] .
$$

On the one hand, we know from Little's law (e.g. Kleinrock 1975) that the average workin-process inventory in a system is related to the average flow time through the system. Hence, in the stochastic system, the average number of process batches $\left(E\left[W I P_{P B}\right]\right)$ will be equal to the throughput rate of process batches $\left(\lambda_{P B}\right)$ times the average process batch flow time $\left(E\left[F_{P B}\right]\right)$. This is the case both for a lot-for-lot policy and for a lot splitting policy:

$$
\begin{aligned}
E\left[W I P_{P B}\right]_{L F L} & =\lambda_{P B} E\left[F_{P B}\right]_{L F L} \\
E\left[W I P_{P B}\right]_{L S} & =\lambda_{P B} E\left[F_{P B}\right]_{L S} .
\end{aligned}
$$

The average inventory holding costs per time unit can be calculated by multiplying this average work-in-process inventory by a specified holding cost per process batch per time

\footnotetext{
${ }^{3}$ See also Langevin et al., 1999 for related research on optimal transfer batch sizing in two-stage deterministic systems.
} 
unit, $c_{i n v}(>0)$ :

$$
\begin{aligned}
E\left[\text { Cost }_{i n v}\right]_{L F L} & =c_{i n v} \lambda_{P B} E\left[F_{P B}\right]_{L F L}, \\
E\left[\text { Cost }_{i n v}\right]_{L S} & =c_{i n v} \lambda_{P B} E\left[F_{P B}\right]_{L S} .
\end{aligned}
$$

As $E\left[F_{P B}\right]$ is convex and continuously decreasing in $T$, the average inventory holding costs per time unit will also be convex and continuously decreasing in $T$.

On the other hand, the average material handling costs per time unit will be linearly increasing in $T$ :

$$
\begin{aligned}
E\left[\text { Cost }_{\text {trans }}\right]_{L F L} & =c_{\text {trans }} \lambda_{P B}, \\
E\left[\text { Cost trans }_{\text {tra }}\right]_{L S} & =c_{\text {trans }} \lambda_{P B} T
\end{aligned}
$$

where $c_{\text {trans }}$ represents a fixed cost per material movement $\left(c_{\text {trans }}>0\right)$.

Consequently, the average total costs will be a convex function of $T$, and an optimal policy will exist for which $E\left[\right.$ Cost $\left._{t o t}\right]$ is minimized. For a system with given cost parameters $c_{i n v}$ and $c_{\text {trans }}$, splitting the process batch in $T$ sublots will be preferable to the lot-for-lot policy from a cost perspective if the following condition is satisfied:

$$
E\left[\operatorname{Cost}_{\text {inv }}\right]_{L F L}>E\left[\operatorname{Cost}_{\text {inv }}\right]_{L S}+(T-1) * \lambda_{P B} * c_{\text {trans }},
$$

which implies

$$
c_{\text {inv }} \Delta E\left[F_{P B}\right]>(T-1) * c_{\text {trans }},
$$

with $\Delta E\left[F_{P B}\right]$ representing the flow time improvement obtained through lot splitting:

$$
\Delta E\left[F_{P B}\right]=E\left[F_{P B}\right]_{L F L}-E\left[F_{P B}\right]_{L S} .
$$

Expression (27) is an intuitive result: the use of lot splitting yields an improvement in total costs only when the realized savings on inventory holding costs counterbalance the increase in material handling costs incurred.

\subsection{Application}

In this section, we apply the cost model to both Case 1 and Case 2 introduced in section 4 (the input parameters for both cases are given in Table 2). Figure 4 shows the obtained flow time improvement $\Delta E\left[F_{P B}\right]$, for the three utilization ratios, at all possible values of $T$ $(T=2,3,5,6,10,15,30)$. The top pane refers to Case 1 , the bottom pane to Case 2 . The 
straight line in both panes refers to $(T-1) c_{\text {trans }} / c_{i n v}$. Without loss of generality, the values of the cost parameters were fixed at $c_{\text {trans }}=2$ and $c_{i n v}=1$. Those values of $T$ for which $\Delta E\left[F_{P B}\right]>(T-1) c_{\text {trans }} / c_{i n v}$ represent cost-efficient lot splitting strategies (see expression (27) above). From this figure, we can derive the following insights:

- At any given utilization rate, an increase in $c_{i n v}$ (or, equivalently, a decrease in $c_{\text {trans }}$ ) favors the use of a larger number of sublots. This is an intuitive result: using a large number of sublots is more attractive in settings where either material handling costs are low, or inventory holding costs are high.

- For given cost parameters $c_{i n v}$ and $c_{\text {trans }}$, the use of a large number of sublots is financially more attractive when the first stage is not the outspoken bottleneck. This is also quite intuitive: in severely unbalanced settings (as in Case 1), the expected flow time improvement which may be obtained through lot splitting is limited. Hence, ceteris paribus, the use of lot splitting is less attractive.

Table 4 gives an overview of the resulting total costs per time unit, in terms of the lot splitting policy $T$. The cost-efficient lot splitting policies are indicated in italics, with the optimal policy given in bold. As an illustration, Figure 5 shows the cost components and the resulting total costs per time unit for both cases, when $\rho_{1}=0.9$. The figure reveals that the total costs per time unit are indeed convex, implying the existence of an optimal policy.

Table 4. Average total costs per time unit for Case 1 and Case 2, when $c_{\text {trans }}=2$ and $c_{i n v}=1$.

\begin{tabular}{l|lll|lll} 
& \multicolumn{3}{|c|}{ Case1 } & \multicolumn{3}{c}{ Case 2} \\
\hline \hline & $\rho_{1}=0.3$ & $\rho_{1}=0.6$ & $\rho_{1}=0.9$ & $\rho_{1}=0.3$ & $\rho_{1}=0.6$ & $\rho_{1}=0.9$ \\
\hline \hline$T=1$ & 0.4380 & 1.1219 & 4.2713 & 0.6993 & 1.7562 & 5.4841 \\
$T=2$ & $\boldsymbol{0 . 4 0 9 9}$ & $\mathbf{1 . 0 6 5 6}$ & $\mathbf{4 . 1 8 6 9}$ & 0.5855 & 1.5269 & 5.1354 \\
$T=3$ & 0.4192 & 1.0842 & 4.2150 & $\mathbf{0 . 5 5 9 0}$ & $\mathbf{1 . 4 7 1 9}$ & 5.0380 \\
$T=5$ & 0.4492 & 1.1441 & 4.3050 & 0.5603 & 1.4737 & $\mathbf{5 . 0 3 6 4}$ \\
$T=6$ & 0.4661 & 1.1779 & 4.3556 & 0.5699 & 1.4927 & 5.0622 \\
$T=10$ & 0.5373 & 1.1779 & 4.5694 & 0.6268 & 1.6061 & 5.2312 \\
$T=15$ & 0.6292 & 1.5041 & 4.8450 & 0.7114 & 1.7752 & 5.4834 \\
$T=30$ & 0.9086 & 2.0628 & 5.6831 & 0.9836 & 2.3194 & 6.2988 \\
\hline
\end{tabular}


Figure 4. $\Delta E\left[F_{P B}\right]$ in terms of the number of sublots $T$, for Case 1 (top pane), and Case 2 (bottom pane). Lot splitting is favorable w.r.t. a lot-for-lot policy only if $\Delta E\left[F_{P B}\right]>$ $(T-1) c_{\text {trans }} / c_{\text {inv }}$.
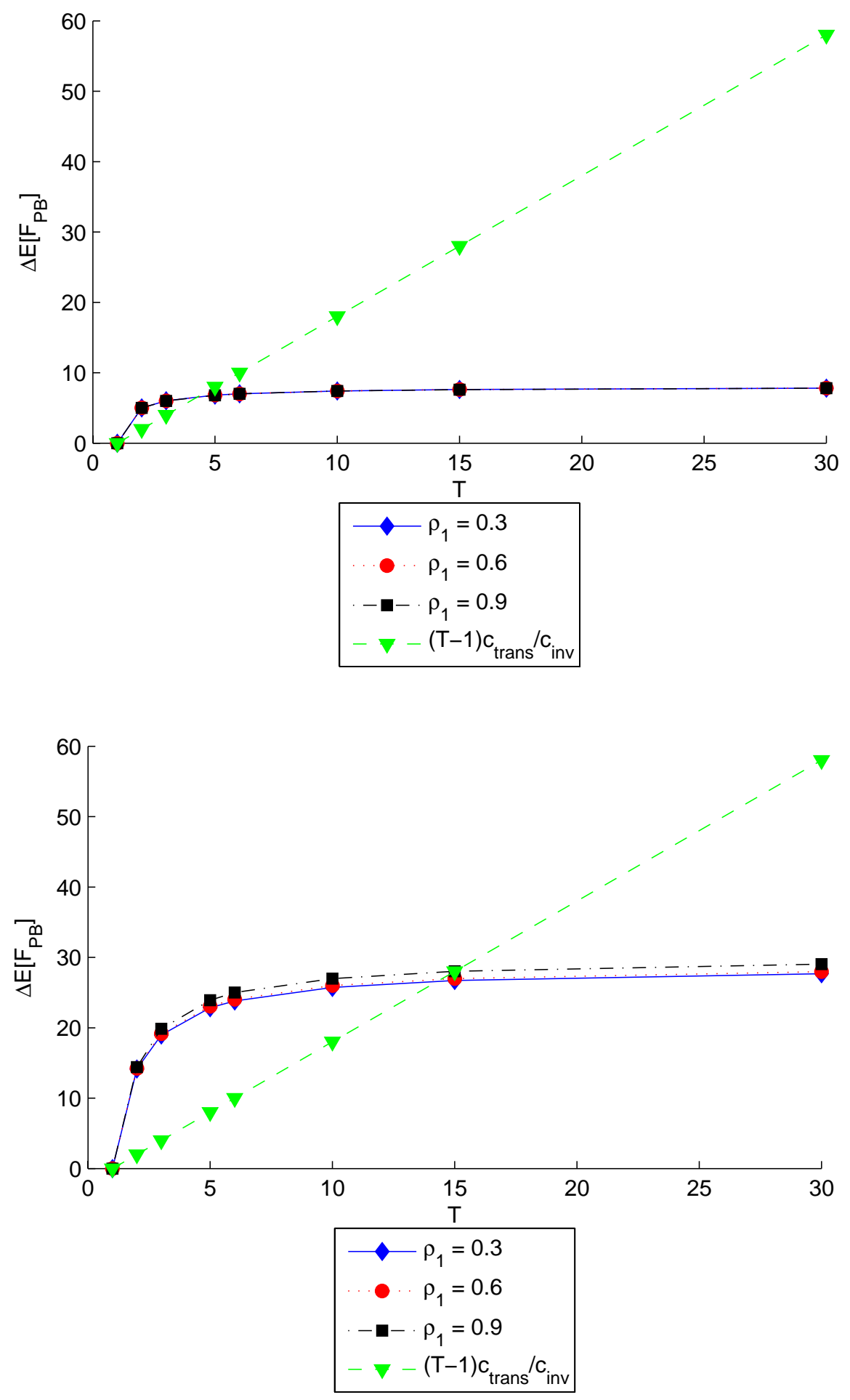
Figure 5. Cost components and resulting total costs per time unit, in terms of the number of sublots $T$, for Case 1 (top pane), and Case 2 (bottom pane), when $\rho_{1}=0.9$.
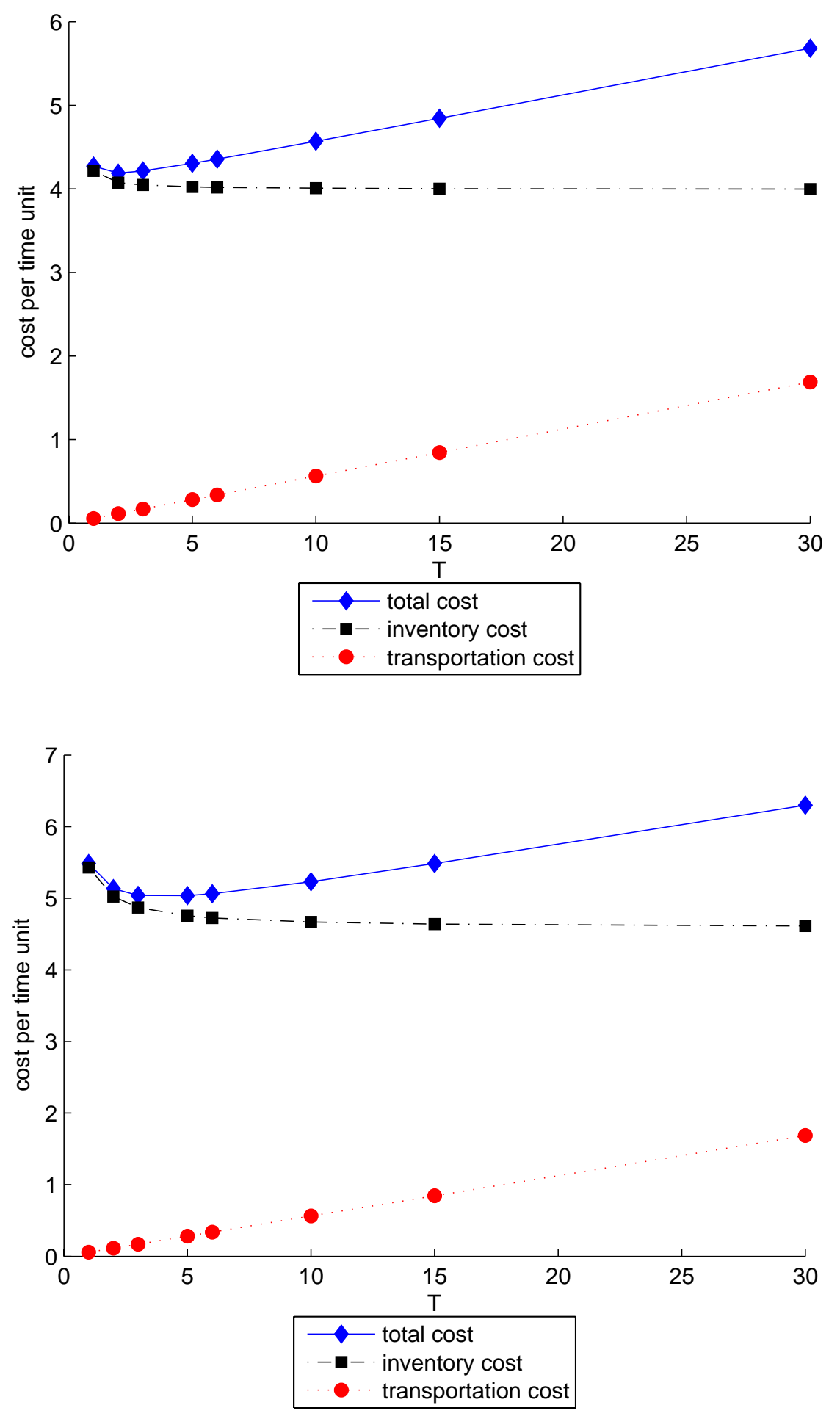


\section{Conclusions}

In this paper, we have studied how the queueing methodology can be applied to estimate average process batch flow times in a stochastic manufacturing system with lot splitting. The discussion has revealed that we have to resort to an embedded queueing model, as the use of lot splitting entails characteristics which prevent the direct application of queueing models to this type of system. This model turns out to yield satisfactory estimates for the average flow time, when compared to simulation results.

The analysis revealed that the use of lot splitting tends to improve the reliability of production run start times at the downstream stage. The relative improvement is most pronounced in settings where the utilization of the upstream stage is low; it disappears when this utilization tends to unity. Similarly, it was shown that the use of lot splitting will likely yield an increase in the variability of process batch makespans on the downstream stage, when the upstream stage is the bottleneck. When the downstream stage is an outspoken bottleneck, this variability effect disappears. When the two stages are closely balanced, the use of lot splitting may actually drive down the variability of process batch makespan on the downstream stage.

It was shown that the flow time improvements in a system with lot splitting come at a price: indeed, the use of lot splitting inevitably leads to more material handling on the shop floor. The presented model provides added value in quantifying the trade-off between material handling costs and inventory holding costs in a system with lot splitting.

Though the setting studied here is limited to a system with two stages and one product family, we believe this work provides an important first contribution, by presenting a generic methodology for modeling the impact of lot splitting in stochastic manufacturing systems. The extension of the model towards more general settings, such as systems with multiple product types and job shop settings, may require additional modifications. Given the frequent use of lot splitting in real-life settings, these extensions undoubtedly provide an interesting and challenging topic for further research.

\section{Appendix 1}

For any zero-inflated normal variable $V^{\prime}=\max [0, V]$ for which $E(V)$ and $\operatorname{Var}(V)$ are known, the first moment $E\left(V^{\prime}\right)$ and second moment $E\left(V^{\prime 2}\right)$ can be determined numerically using 
the following expressions (Blumenfeld, 2001):

$$
\begin{gathered}
E\left(V^{\prime}\right)=\frac{E(V)}{2}\left(1+\operatorname{Erf}\left[\frac{E(V)}{\sqrt{2 \operatorname{Var}(V)}}\right]\right)+\operatorname{Exp}\left\{\frac{-E(V)^{2}}{2 \operatorname{Var}(V)}\right\} \sqrt{\frac{\operatorname{Var}(V)}{2 \pi}}, \\
E\left(V^{\prime 2}\right)=\operatorname{Exp}\left\{\frac{-E(V)^{2}}{2 \operatorname{Var}(V)}\right\} E(V) \sqrt{\frac{\operatorname{Var}(V)}{2 \pi}}+\frac{E(V)^{2}+\operatorname{Var}(V)}{2}\left(1+\operatorname{Erf}\left[\frac{E(V)}{\sqrt{2 \operatorname{Var}(V)}}\right]\right) .
\end{gathered}
$$

The variance of $V^{\prime}$ then follows immediately from:

$$
\operatorname{Var}\left(V^{\prime}\right)=E\left(V^{\prime 2}\right)-\left[E\left(V^{\prime}\right)\right]^{2}
$$

The notation $\operatorname{Er} f[x]$ in these expressions refers to the error function in $x$ :

$$
\operatorname{Er} f[x]=\frac{2}{\sqrt{\pi}} \int_{0}^{x} \exp \left\{-t^{2}\right\} d t .
$$

\section{Appendix 2}

In general, for any two correlated normal random variables $U$ and $Z, \operatorname{Cov}(U, \max [Z, 0])$ can be numerically determined as follows:

$\operatorname{Cov}(U, \max [Z, 0])$

$=E(U \max [Z, 0])-E(U) E(\max [Z, 0])$

$=\int_{-\infty}^{+\infty} \int_{0}^{+\infty} u * z * f_{U, Z}(u, z) * d z d u-E(U) E(\max [Z, 0])$

The notation $f_{U, Z}(u, z)$ denotes the bivariate normal density function for the two correlated variables $U$ and $Z$ :

$f_{U, Z}(u, z)=\left(2 \pi \sigma_{U} \sigma_{Z} \sqrt{1-\rho_{U, Z}^{2}}\right)^{-1} h(u, z)$,

$h(u, z)=\operatorname{Exp}\left\{\frac{-1}{2\left(1-\rho_{U, Z}^{2}\right)}\right\}\left[\frac{(u-E(U))^{2}}{\operatorname{Var}(U)}-2 \rho_{U, Z} \frac{(u-E(U))}{\sigma_{U}} \frac{(z-E(Z))}{\sigma_{Z}}+\frac{(z-E(Z))^{2}}{\operatorname{Var}(Z)}\right]$,

in which $\rho_{U, Z}$ refers to the correlation coefficient of $\mathrm{U}$ and Z. The expression for $E(\max [Z, 0])$ is given in Appendix 1. Consequently, $\operatorname{Cov}(U, \max [Z, 0])$ can be numerically determined for any two normal random variables $U$ and $Z$ as long as $E(U), E(Z), \operatorname{Var}(U), \operatorname{Var}(Z)$ and $\rho_{U, Z}$ are known. 


\section{Acknowledgments}

This research was supported by the Research Foundation Flanders (Belgium). At the time of writing, Ms Van Nieuwenhuyse was a Postdoctoral Fellow of the Research Foundation Flanders. 


\section{References}

Baker, K.R. and Jia, D., "A Comparative Study of Lot Streaming Techniques", Omega, Vol. 21, pp. 561-566 (1993).

Benjaafar, S., "On Production Batches, Transfer Batches, and Lead Times", IIE Transactions, Vol. 28, pp. 357-362 (1996).

Blumenfeld, D., Operations Research Calculations Handbook, CRC Press, Boca Raton, FL (2001).

Bozer, Y.A. and Kim, J., "Determining Transfer Batch Sizes in Trip-based Material Handling Systems", International Journal of Flexible Manufacturing Systems, Vol. 8, No. 4, pp. 313-356 (1996).

Bukchin, J., Tzur, M., and Jaffe, M., "Lot Splitting to Minimize Average Flow Time in a Two-Machine Flow Shop", IIE Transactions, Vol. 34, pp. 953-970 (2002).

Buzacott, J.A. and Shantikumar, J.G., "On Approximate Queueing Models of dynamic Job Shops", Management Science, Vol. 31, pp. 870-887 (1985).

Chen, J. and Steiner, G., "Lot Streaming with Attached Setups in Three-Machine Flowshops", IIE Transactions, Vol. 30, pp. 1075-1084 (1998).

Goldratt, E.M. and Cox, J., The Goal: a Process of Ongoing Improvement, North River Press, New York, NY (1984).

Graves, S.C. and Kostreva, M.M., "Overlapping Operations in Materials Requirements Planning", Journal of Operations Management, Vol. 6, pp. 283-294 (1986).

Hopp, W.J., Spearman, M.L., and Woodruff, D.L., "Practical Strategies for Lead Time Reduction", Manufacturing Review, Vol. 3, pp. 78-84 (1990).

Hopp, W. J. and Spearman, M.L., Factory Physics: Foundations of Manufacturing Management, Irwin/McGraw-Hill, New York, NY (2000).

Jacobs, F.R. and Bragg, D.J., "Repetitive Lots: Flow-Time Reductions through Sequencing and Dynamic Batch Sizing", Decision Sciences, Vol. 19, pp.281-294 (1988).

Karmarkar, U., Kekre, S., and Freeman, S., "Lot Sizing and Lead Time Performance in a Manufacturing Cell", Interfaces, Vol. 15, pp. 1-9 (1985a).

Karmarkar, U., Kekre, S., and Kekre, S., "Lot Sizing in Multi-Item Multi-Machine Job 
Shops", IIE Transactions, Vol. 17, pp. 290-298 (1985b).

Karmarkar, U., "Lot Sizes, Lead Times and In-Process Inventories", Management Science, Vol. 33, pp. 409-417 (1987).

Kleinrock, L., Queueing Systems, John Wiley \& Sons, New York, NY (1975).

Kramer, W. and Lagenbach-Belz, M. "Approximate Formulae for the Delay in the Queueing System GI/GI/1", pp. 2351-2358 in The Congressbook of the Eight International Teletraffic Congress, Melbourne (1976).

Kropp, D.H. and Smunt, T.L., "Optimal and Heuristic Models for Lot Splitting in a Flow Shop", Decision Sciences, Vol. 21, pp. 691-709 (1990).

Lambrecht, M.R., Ivens, P.L. and Vandaele, N.J., "ACLIPS: A Capacity and Lead Time Integrated Procedure for Scheduling", Management Science, Vol. 44, pp. 1548-1561 (1998).

Langevin, A., Riopel, D. and Stecke, K.E., "Transfer Batch Sizing in Flexible Manufacturing Systems", Journal of Manufacturing Systems, Vol. 18, pp. 140-151 (1999).

Litchfield, J.L. and Narasimhan, R., "Improving Job Shop Performance through Process Queue Management under Transfer Batching", Production and Operations Management, Vol. 9, pp. 336-348 (2000).

Marshall, K.T., "Some Inequalities in Queueing", Operations Research, Vol. 16, pp. 651-665 (1968).

Potts, C.N. and Kovalyov, M.Y., "Scheduling with Batching: a Review", European Journal of Operational Research, Vol. 120, pp. 228-249 (2000).

Ramasesh, R.V., Fu, H., Fong, D.K.H. and Hayya, J.C., "Lot Streaming in Multistage Production Systems", International Journal of Production Economics, Vol. 66, pp. 199$211(2000)$.

Ruben, R.A. and Mahmoodi, F., "Lot Splitting in Unbalanced Production Systems", Decision Sciences, Vol. 29, pp. 921-949 (1998).

Santos, C. and Magazine, M., "Batching in Single-Operations Manufacturing Systems", Operations Research Letters, Vol. 4, pp. 99-103 (1985).

Smunt, T.L., Buss, A.H. and Kropp, D.H., "Lot Splitting in Stochastic Flow Shop and Job Shop Environments", Decision Sciences, Vol. 27, pp. 215-238 (1996). 
Suri, R., Quick Response Manufacturing: a Companywide Approach to Reducing Lead Times, Productivity Press, Portland, OR (1998).

Suri, R., Sanders, J.L. and Kamath, M., "Performance Evaluation of Production Networks", pp. 199-286 in Logistics of Production and Inventory, Handbooks in Operations Research and Management Science, S. C. Graves et al. (Eds), North-Holland (1993).

Umble, M. and Srikanth, M.L., Synchronous Manufacturing: Principles for World-Class Excellence, The Spectrum Publishing Company, Wallingford, CT (1995).

Vandaele, N. J., Van Nieuwenhuyse , I. and Cupers, S., "Optimal Grouping for a Nuclear Magnetic Resonance Scanner", European Journal of Operational Research, Vol. 151, pp. 181-192 (2003).

Van Nieuwenhuyse, I. Lot splitting in single-product flowshops: issues of delivery reliability, production disruptions and flow times. PhD thesis, Department of Applied Economics, University of Antwerp, Antwerp, Belgium (2004).

Van Nieuwenhuyse, I. and Vandaele, N., "Determining the Optimal Number of Sublots in a Single-Product, Deterministic Flow Shop with Overlapping Operations", International Journal of Production Economics, Vol. 92, pp. 221-239 (2004a).

Van Nieuwenhuyse, I. and Vandaele, N., "The Impact of Delivery Frequency on Delivery Reliability in a Two-Stage Supply Chain", Proceedings of the 13th International Workshop on Production Economics, Vol. 3, pp. 367-392 (2004b).

Van Nieuwenhuyse, I. and Vandaele, N., "The Impact of Delivery Lot Splitting on Delivery Reliability in a Two-Stage Supply Chain", International Journal of Production Economics, forthcoming (2006).

Van Nieuwenhuyse, I. and Vandaele, N., "Analysis of Gap Times in a Two-Stage Stochastic Flowshop with Overlapping Operations". Department of Applied Economics, University of Antwerp, Research paper 2005-004 (2005).

Wagner, B.J. and Ragatz, G.L., "The Impact of Lot Splitting on Due Date Performance", Journal of Operations Management, Vol. 12, pp. 13-25 (1994).

Whitt, W., "The Queueing Network Analyzer", The Bell System Technical Journal, Vol. 62, pp. 2779-2815 (1983).

Whitt, W., "Approximations for the GI/G/m Queue", Production and Operations Management, Vol. 2, pp. 114-161 (1993). 
Whitt, W., "Towards Better Multi-Class Parametric-Decomposition Approximations for Open Queueing Networks", Annals of Operations Research, Vol. 48, pp. 221-248 (1994). 